\title{
Is recognition accuracy really impaired when the target is repeated in the display?
}

\author{
CHARLES W. ERIKSEN, NANCY MORRIS, YEI-YU YEH, \\ WILLIAM O'HARA, and ROBERT T. DURST \\ University of Illinois, Champaign-Urbana, Champaign, Illinois 61820
}

\begin{abstract}
Bjork and Murray (1977) have presented a feature-specific interactive channels model that predicts perceptual interference between letters presented simultaneously in visual displays. Maximum interference is predicted when a target letter is presented with an identical letter. In their experiment, Bjork and Murray found support for their model, but their results could have occurred artifactually from response biases of their subjects. Santee and Egeth (1980) used a different paradigm that eliminated the possibility of this particular response bias and roported that their data supported the feature-specific model. However, the present paper shows that analysis of their data by Santee and Egeth was incomplete and the comparisons inappropriate. In two experiments that used the Santee and Egeth paradigm, we failed to find support for the featurespecific model when more detailed analyses of the data were undertaken. In a third experiment, one that used the Bjork and Murray paradigm, but with control of response bias, no significant or suggestive evidence was found that feature similarity between noise and target letters affected recognition of the latter.
\end{abstract}

Search for a target letter in a matrix or display of letters or symbols is a relatively complex task, and it is not unexpected that performance should involve a number of processes and variables (see Eriksen \& Schultz, 1979, for a review). Recently Bjork and Murray (1977) have proposed that some of the interference produced by noise items in the display arises at an early perceptual stage of processing. In an extension of Estes' (1972) interactive channels model, they assume that alphanumeric characters are represented in memory and in the perceptual system by sets of features. Information from features in a visual stimulus is extracted by parallel interactive input channels leading to feature detectors. Input channels leading to feature detectors necessary for target items are assumed to be in a heightened state of excitability, and thus are more readily activated. Excitation of an input channel results in inhibition of other channels leading to the same feature detector and a generalized inhibition of all other input channels. Since interference is a result of excitation of input channels leading to common feature detectors, a noise item interferes with detection of a target item to the extent that they share common features. A noise item identical to the target should interfere the most, since it would have a complete overlap of features with the target.

In their experiment, Bjork and Murray (1977) employed a recognition paradigm in which one or two

This research was supported by Public Health Service Research Career Program Award K6-MH-22014 to the first author and by USPHS Research Grant MH-01206. letters appeared in a $4 \times 4$ matrix of number signs (\#). Stimulus displays were preceded by a $4 \times 4$ matrix of dollar signs (\$) and followed immediately by the same dollar-sign matrix plus an upward-pointing arrow beneath one of the columns. The target displays were exposed for short durations so as to yield accuracy of approximately $75 \%$. The subject responded by saying which of two letters, B or R, had appeared in the column indicated by the arrow. There were four types of stimulus displays: (1) single target - the display contained only one letter, B or R; (2) noise same as target-the display contained either two Bs or two Rs; (3) noise alternative targetboth $B$ and $R$ were present; and (4) noise nontargeteither $\mathbf{B}$ or $\mathbf{R}$ was present with a nontarget letter, $\mathbf{P}$ or $\mathbf{K}$.

Bjork and Murray derived several predictions from the feature-specific inhibitory channels model as to relative performance on these displays. The more important of these predictions were that recognition performance on single-target displays should be superior to recognition on other display types, and that performance on noise-alternative-target displays should be superior to performance on noise-same-as-target displays. The results confirmed these predictions. Average performance on single-target displays was $90 \%$ correct as compared with $74 \%$ for noise same as target and $84 \%$ for noise alternative target.

While these results are rather well described by the Bjork and Murray feature-specific inhibitory channels model, they are, nonetheless, surprising. They are in apparent conflict with a substantial body of experimental data that indicate that perceptual processing of simultaneously presented forms is inde- 
pendent (Eriksen \& Lappin, 1965, 1967; Eriksen \& Spencer, 1969; Gardner, 1973; Schiffrin \& Geisler, 1973). The most direct conflict is with a series of experiments that addressed specifically the effect of target redundancy upon the identification of tachistoscopically presented letter forms. In the Eriksen and Lappin experiment (1965), the displays contained from one to six occurrences of the same target letter. The data were well described by a model of perceptual independence that assumed that each occurrence of the target represented an independent opportunity for the subject to perceive it. The independence model was confirmed by Eriksen (1966), with displays containing two repetitions of the target letter, and by Eriksen, Greenspon, Lappin, and Carlson (1966) and Eriksen and Greenspon (1968) for binocular, dichoptic, and monocular displays.

These experiments differed from the Bjork and Murray experiment in that the subject was aware that all letters in the display were identical. Thus he could take advantage of the independent opportunities to perceive. It might be argued that the opportunity the subject had to use the redundancy counteracted or offset recognition impairment due to inhibition among features. However, if the type of interference specified by Bjork and Murray was occurring, the obtained performance could not be at the level to be expected from a model of independence. In the redundancy studies cited above, the fit of the obtained data to the prediction from the independence model was within one or two percentage points, with the exception of the Eriksen and Lappin (1965) study, in which the obtained data were at a higher level of recognition accuracy for four and six target repetitions than predicted by independent chances.

Santee and Egeth (1980) used $A$ and $E$ as target letters and replicated part of the Bjork and Murray results. They found that performance was indeed better for the noise-alternative-target displays than for noise-same-as-target displays, but performance for single-target displays was significantly poorer than it was for either of the other two display types. They attributed the poorer performance for the single displays to interference from the pre- and postmasking fields. They noted, however, that their results, as well as those of Bjork and Murray, could be due to the operation of a response bias. Santee and Egeth state: "First, assume that on some trials one of the letters in the double letter displays is not seen clearly, and than on approximately half of these trials, the unclear letter is in the cued column. Second, assume that the subject has a bias to respond to a cued but undifferentiated letter with the name of the target letter opposite to that of the one seen clearly. For example, if the subject sees an $A$ and a cued but unclear letter, then he or she would tend to respond "E." It follows that accuracy would be lower when the noise (i.e., uncued) letter was the same as the target (as in the AA condition) than when the noise letter was the alternative target (as in the $\mathrm{AE}$ condition). Thus, performance in the AA and AE conditions might be an artifact of response bias rather than the result of perceptual interference" (Santee \& Egeth, 1980 , p. 324).

The possibility of this response bias brings into serious question the validity of the Bjork and Murray model, particularly since the experimental situation in both the Bjork and Murray study and the Santee and Egeth replication was such as to teach a subject just such a response bias. In the Bjork and Murray paradigm, a target letter (B or $\mathbf{R}$ ) occurs on every trial, and the subject is required to discriminate between these two letters. An efficient strategy that a subject could adopt would be to search for only one of the letters, say, the B. Following a trial, if his criterion for $B$ is reached or exceeded he responds "B." If the criterion is not reached, he says $R$. He reduces the task to a signal in noise detection. Now consider the subject during the practice session and in the early trials during the experimental sessions. He's looking for a B. On a certain proportion of the trials, he sees both target letters. The attentive subject will note that two-thirds of the time when he sees a B and there is a second target letter in the display, the other target is an $\mathbf{R}$. This situation occurs because the four possible two target displays (BB, RR, BR, RB) are used with equal frequency. Thus, if the subject sees a B, there is only one display alternative that contains a second $B$ (BB) but two equally likely alternatives (BR and RB) that contain an $R$ as the second letter. The tendency for subjects to probability match is well established (Humphreys, 1939), and it seems unreasonable to assume that most subjects are sophisticated enough in assessing probabilities to realize that, when the position of the B is specified (right or left), the probability of $\mathbf{R}$ as the second target reduces to one-half. ${ }^{1}$ The apparent probability that the subject has observed during the practice and early experimental sessions may well result in his adopting a strategy of probability matching and thus increasing hits on alternative target displays and decreasing hits on identical displays.

Neither Bjork and Murray nor Santee and Egeth are aware, apparently, of the extent to which their use of equal frequency for the different target display types could have built a response bias into their subjects. However, Santee and Egeth advance two arguments against a response-bias interpretation of their experiment and the data of Bjork and Murray. Santee and Egeth argue that the noise-nontarget condition (AK EK, etc., in their study) is a built-in control for response bias. Presumably, if performance in the alternative-target condition was inflated due to the subject's bias to guess the alternative letter on those trials in which only one target is seen, then performance on the alternative condition would be 
higher than on the noise-nontarget condition. In the absence of response bias, performance in these conditions should be equal, according to the featurespecific inhibition model, since the designated target is in both cases paired with a noninterfering letter. Since performance was equal in both the Bjork and Murray experiment and in the replication, Santee and Egeth consider this strong evidence that the response bias did not inflate the proportion of correct responses in the alternative target condition.

This argument, however, is not persuasive. The noise-nontarget condition would be a suitable control were it not for the use of a postdisplay cue that designated the letter to be reported. It has been shown that several hundred milliseconds can be required to perceive a cue and select the designated alternative (Averbach \& Corriell, 1961; Colegate, Hoffman, \& Eriksen, 1973). During this time, the information received from the alternative-target display is subject to decay. It is likely that the subject does not wait for the processing of the cue before he begins encoding one of the letters, but half of the time this will turn out to be the letter that is not subsequently cued. Thus, he then will have to decipher the decayed trace or icon of the cued letter. The noise-nontarget condition differs from the alternativetarget condition in this important respect-the nontarget noise letter can serve as an early cue. If the subject begins encoding the nontarget letter first, as soon as he recognizes it as not being an $\mathbf{A}$ or $\mathbf{E}$, he can shift processing to the target. This could occur before the postdisplay cue is finished processing, and thus the target would be attended sooner. Research has shown that an appreciable gain in recognition performance in multielement displays is achieved when the cue precedes the display by as little as $\mathbf{5 0} \mathrm{msec}$ (Eriksen \& Collins, 1969; Eriksen \& Rohrbaugh, 1970). The gain from earlier cuing in the noise-nontarget condition may be enough to offset the performance gain in the alternative-target condition that occurs from response biases.

Santee and Egeth provide a second argument against the response bias interpretation based upon the results from two briefly described experiments. In one experiment, the identical and the alternative target conditions were compared with the condition in which a target letter appeared with a nontarget $O$ (e.g., AO, EO). Both the target and the $O$ were cued equally often by the postdisplay arrow. The other experiment was identical, except that $O$ was replaced by an illegible form made by overstriking an $A$ with an $E$ and in some cases also overstriking this composite with a number sign. Santee and Egeth argue that the response bias hypothesis would require that the subjects respond to the cued nontarget letter with the name of the target letter not presented on that trial. Since there was a slight tendency for the subjects to respond with the target that was present,
Santee and Egeth conclude that the results suggest that response bias cannot account for the results of Bjork and Murray or the replication.

Again, however, these two control experiments are not quite apt. On a significant proportion of the trials, the subject can be expected to perceive both the target letter and the $O$. Consider the subject's dilemma on such a trial. He perceives an $A$ and $O$, but the cue designates the $O$. Since he can respond only by saying $A$ or $E$, he is in a quandary. He has been instructed that only $A$ and $E$ are targets, so he decides he was mistaken as to which position the $\mathbf{A}$ had occupied. Thus, he responds $\mathbf{A}$. This effect would offset a bias to respond with the nonpresented target letter on those trials on which he perceives only the $A$, and the $O$ location is subsequently cued.

Apparently, Santee and Egeth also were less than satisfied with these arguments, since they devised a different experimental paradigm to eliminate the possibility of response bias. In their paradigm, no singleletter displays are employed. Instead all displays contain two letters. In the identical-target displays, the target was repeated (AA or EE). In the alternativetarget displays, both targets occurred (AE, EA). In a third condition, a target occurred with a nontarget noise letter ( $K$ or $L)$. The subject's task was to say "yes" if he detected either an A or an E in the display. Targets were present on only half of the trials, with the remaining trials consisting of noise displays composed of the two nontarget letters $K$ and $L$.

With this paradigm, Santee and Egeth (Experiment 3 ) found that the averaged performance on alternative-target displays was superior to the averaged performance on identical-target displays, the proportion of yes responses being .86 and .77, respectively. Performance on displays containing a target with a nontarget noise letter was . 71 , and the false-alarm rate (yes responses to the permutations of $K$ and $L$ ) was . 30 .

While this new paradigm eliminates the kind of response bias that may have plagued the experiment of Bjork and Murray and the Santee and Egeth replication, the analysis of the results is subject to an equally serious artifact. The comparison of averaged performance on identical-target and alternativetarget displays requires that the target letters $\mathbf{A}$ and $E$ be exactly equal in discriminability in the experimental context. To illustrate, let us assume that the recognition accuracy for $A$ among the experimentally presented alternatives is .90 and that for $E$ is .70 . Let us further assume the simplest null hypothesis, namely that the identifiability of these two letters is unaffected by pairing with another in the display and that, with a compound display such as $\mathrm{AE}$, accuracy is determined by the letter most readily perceived, namely the A. Thus, in our illustration, AA displays would have a proportion correct of .90 and $\mathrm{EE}$ displays .70 . But, if we averaged the performance on 
AA and EE displays as Santee and Egeth did, we would obtain an average proportion correct of .80 . But $\mathrm{AE}$ and EA displays contain an $\mathbf{A}$, which gives both of them a recognition accuracy of .90 , and thus we get a superiority for these displays that is nothing more than the result of arithmetic averaging. The logic of this analysis is essentially the same as that employed by Biederman and Checkosky (1970) and Garner (1969) in comparing the discriminability of compounded stimuli with stimuli differing on the single dimensions that were constituents of the compound.

Santee and Egeth would have been able to eliminate the above interpretation of their data had they shown that alternative-target displays were significantly better than either AA or EE displays. But this still would not have made their results clear and unequivocal support for the feature-specific model. The failure of their paradigm to provide information on the hit rate for single targets renders the paradigm incapable of providing a clear test of the featurespecific model. The model makes the prediction that perception of repeated letter displays is impaired, but the prediction of impairment requires a criterion of what unimpaired performance should be. In the Bjork and Murray experiment, this criterion was the recognition for a target letter presented alone. But Santee and Egeth's Experiment 3 provides no criterion as to what the performance level should be for unimpaired recognition. Even if they had found that the alternative-target condition was significantly superior to both $A A$ and $E E$ conditions, this would not necessarily imply that AA and EE were impaired. Instead, the conclusion might be that a diversification of the perceptual input improves recognition performance. This is not a trivial point. If it were found that repeated targets were not impaired but alternative targets showed a facilitation, the theoretical implications would be important and quite different from explanations trying to account for impairment.

There are several reasons why alternative target displays might show superior recognition accuracy relative to repeated target displays. Since the letters are presented side by side in rather close proximity, the effect might be due to such pedestrian variables as differences in contour interactions or to differences in digram frequency which were not controlled. One might be a bit more imaginative and posit that at low stimulus energies, recognition processes are facilitated or primed by more diverse inputs. Indeed, such speculation regarding an alternative letter superiority effect is encouraged by Egeth and Santee (1981), who in another paper have reported that substitution of the lowercase version for one of the letters in identical-target and alternative-target displays yields a pattern of results that is the same as that obtained by Bjork and Murray. In other words, Ae and eA displays show higher recognition than do Aa and Ee displays when $A$ and $E$ are targets. Due to the marked differences in features between the upperand lowercase version of the target letters, it would be exceedingly difficult to explain the results in terms of feature-specific inhibition.

\section{EXPERIMENTS 1 AND 2}

At this point, the "repeated-letter inferiority effect," as Egeth and Santee (1981) have termed it, is at best questionable. Not only is the effect at variance with the redundant target experiment we have reviewed above, but also the Bjork and Murray paradigm has been seen to be subject to an unassessed responsebias effect and the results from the Santee and Egeth paradigm are inconclusive due to incomplete data presentation and analysis. The Santee and Egeth paradigm, however, does have the merit of avoiding the kind of response bias that may have operated in the other paradigm. What seems to be needed is further experimentation using the Santee and Egeth paradigm but with an assessment of the discriminability of the individual target letters and a more detailed analysis of the data.

Experiment 1 was quite similar to Santee and Egeth's Experiment 3, with the exception that the procedure was modified to include single-target conditions. Like the Santee and Egeth experiment, preand postmasking fields were employed. Experiment 2 differed from Experiment 1 only in that it eliminated the pre- and postmasking fields. We considered it important to assess what effect pre- and postmasking stimuli were contributing to the experimental effects. Eriksen (1980) has pointed out some of the methodological problems that are involved in using visual masks, particularly when the experiment involves the differential stimulation of hypothesized feature detectors. The features contained in the mask have an unspecified overlap with the features in the experimental displays. Thus, they pose a source of confounding. This confounding could be rather serious with respect to the feature-specific model, in view of Gilinsky's evidence (1968) that detectors for vertical and horizontal lines can be reduced in sensitivity by preadapting the eye to either the vertical or the horizontal lines before exposing the test stimulus. On the basis of her data, one might wonder to what extent relevant feature detectors are being adapted by the premask.

\section{Method}

Subjects. Seven different subjects were used in each of the two experiments. In Experiment 1, three of the subjects were women, and in Experiment 2, four were women. All were students at the University of Illinois, Urbana-Champaign, and they served as paid volunteers. All had normal or corrected-to-normal vision. 
Apparatus. In Experiment 1, all displays were presented in two channels of a three-channel Scientific Prototype tachistoscope at a luminance of $1.37 \mathrm{~cd} / \mathrm{m}^{2}$, as measured by a Photo Research Corporation Spectra brightness spot meter. Stimulus displays consisted of one or two black paratype Helvetica medium letters against a white vinyl background. The width and height of these letters subtended visual angles of .15 and .20 deg, respectively. In the two-letter displays, letters occupied side-by-side positions in the center of the visual field with a separation of $.22 \mathrm{deg}$ of visual angle. Letters in the single-letter displays occupied either the left or the right position. A fixation display consisted of two dollar signs in each target position, of the same font and size as the target letters.

The letters $A, E, K$, and $L$ appeared in three kinds of displays: single letter (e.g, A, K), two identical letters (e.g., EE, LL), and two different letters (e.g., AL, EA). All possible combinations of letters and display positions were used. Since the letters $A$ and $E$ were targets, there were four types of target displays: single target $(A, E)$, double target (AA, EE), alternative targets (AE, EA), and target/nontarget (e.g., AK, LE).

The equipment and displays were identical for Experiment 2, with the exception that the pre and post dollar-sign masking stimuli in the fixation display were replaced by two paratype hyphens, $.10 \mathrm{deg}$ in length, located below each letter position.

Procedure. In both experiments, subjects served two 45-min sessions. Each session consisted of 32 practice trials and six blocks of 32 experimental trials. Within each block of trials, target and no-target displays occurred with equal probability. There was an equal number of each type of target display and no-target display. The order of presentation in the displays was determined pseudorandomly.

The subjects were instructed to say "yes" if either an A or an E was present in the display and "no" if neither was present. On a verbal cue from the experimenter, a subject initiated a trial by pressing a hand-held switch that caused the fixation display to be replaced by an experimental display for a brief duration. Exposure duration for each subject was adjusted during practice and, if necessary, after each block of trials in order to maintain an overall response accuracy rate of approximately $75 \%$. In Experiment 1 , which employed the visual pre- and postmasks, exposure durations across subjects ranged from 65 to $120 \mathrm{msec}$, with a mean of $88 \mathrm{msec}$. In Experiment 2, exposure durations ranged from 16 to $55 \mathrm{msec}$ with a mean of $35 \mathrm{msec}$.

\section{Results and Discussion}

The data from both experiments are initially presented in the same manner as that employed by Santee and Egeth and are shown in Table 1. Recall that the subject was to say "yes" if he saw either an $A$ or an E, or both of these letters, in a display. The data in Table 1 show the averaged proportion of "yes" responses for identical displays, alternativetarget displays, and displays in which a target letter

Table 1

Mean Proportion of "Yes" Responses to the Three Types of Target Displays in Experiments 1 and 2

\begin{tabular}{lccc}
\hline & \multicolumn{3}{c}{ Experiment } \\
\cline { 2 - 4 } \multicolumn{1}{c}{ Display Type } & 1 & 2 & $(3)$ \\
\hline Identical Target (AA and EE) & .80 & .87 & $(.77)$ \\
Alternative Target (AE and EA) & .91 & .90 & $(.86)$ \\
Target/Nontarget (AK, EK, etc.) & .71 & .72 & $(.71)$ \\
\hline
\end{tabular}

Note-Data from the Santee and Egeth (1980) Experiment 3 are shown in parentheses.
Table 2

Mean Proportion of "Yes" Responses to Each Target Display in Experiments 1 and 2

\begin{tabular}{cccrrrrrr}
\hline $\begin{array}{c}\text { Exper- } \\
\text { iment }\end{array}$ & A & E & AA & EE & AE & EA & $\begin{array}{r}\text { A }+ \\
\text { NTL }\end{array}$ & $\begin{array}{r}\text { ET }+ \\
\text { NTL }\end{array}$ \\
\hline 1 & .91 & .60 & .90 & .71 & .95 & .85 & .82 & .60 \\
2 & .80 & .55 & .93 & .81 & .91 & .90 & .84 & .60 \\
\hline
\end{tabular}

Note $-N T L=$ nontarget letter.

was paired with a nontarget letter (target/nontarget). The values in parentheses are data from Santee and Egeth's Experiment 3, which are provided for comparison.

In Experiment 1, in which we used a mask, as did Santee and Egeth, the results are quite comparable to those they obtained. The averaged performance on the alternative-target displays is superior to the averaged performance on the identical-target displays $(\mathrm{p}<.01)$. Performance on the target/nontarget displays is significantly worse than either identical or alternative-target displays. In Experiment 2, which did not employ a masking stimulus, the pattern of results is similar, but in that instance the difference in performance on alternative-target displays relative to identical-target displays did not approach significance. However, averaged performance for both identical and alternative-target displays was significantly superior to target/nontarget displays $(p<.01)$

As we pointed out in the introduction, these analyses alone are incapable of providing definitive information relative to the feature-specific model. We are now in a position to illustrate these arguments by a more detailed analysis of performance on the specific display types. In Table 2, we have presented the proportion of "yes" responses to the different single-target displays, to the different identical-target displays, and to the two alternative-target displays, as well as the target/nontarget displays for $A$ and E targets.

Consider, first, the data from Experiment 1. The proportion of correct "yes" responses to A and E is seen to differ markedly not only for the singletarget displays, but also for the identical-target displays. The data from this experiment are perfectly consistent with the simple hypothesis that states that the discriminability of a letter is unaffected when paired with another letter and that the discriminability of a compound is the same as the most discriminable member of the pair. The target $A$, when presented alone, yields a proportion correct of .91 . This value does not differ significantly or appreciably from the .90 averaged performance on the alternativetarget displays (AE and EA).

Upon initial examination, the data from Experiment 2 do not seem to be consistent with the simple hypothesis that the discriminability of the com- 
pounded stimulus is determined by the most discriminable member. Although target $A$ is again found to be significantly $(p<.01)$ more discriminable on single-letter displays than the target $\mathrm{E}$, performance on both alternative target displays (AE and EA) is significantly better $(p<.01)$ than it is for $A$ alone. This discrepancy between the results of Experiments 1 and 2 can be understood if we examine the false-alarm rates for one- and two-letter displays between the two experiments. These false-alarm rates are shown in Table 3. In Experiment 1, the falsealarm rate for displays consisting of one noise letter was .25 and for displays of two noise letters, .28 . On the other hand, in Experiment 2 the false-alarm rate for one- and two-letter displays was significantly different $(\mathrm{p}<.01)$.

In Experiment 2, the subjects were more willing to report a target present when two-letter displays were presented than when the displays contained only one letter. The absence of the masking stimulus in Experiment 2 may have permitted the subjects to detect the target more readily when two letters, rather than one, were presented in a display. If the subjects had a response bias to say "yes" when two letters were present, it would account for a differential falsealarm rate for the two-letter displays between the mask and nonmask experiments.

Irrespective of the reason for the higher falsealarm rate in Experiment 2, the greater tendency to say "yes" to two-letter displays acts to inflate the hit rate for identical- and alternative-letter displays. It makes it impossible to compare performance for single-letter displays with the identical- and alternativetarget displays in terms of proportions correct. The difference of .10 in proportion of false alarms between one- and two-letter displays is almost exactly the difference between performance on the singleletter display and performance on the alternativetarget displays (AE and EA). Thus, the data from Experiment 2 can in no way be considered as inconsistent with the simple hypothesis that the discriminability of a compound stimulus is that of the most discriminable member in the pair.

The only data in Experiments 1 and 2 that directly reflect upon the feature-specific model are the data on the single-target displays and the target/nontarget displays. There is a certain amount of feature overlap of the target letters $A$ and $E$ with the noise letters

Table 3

Proportion of False Alarms ("Yes" Responses to Noise-Only Displays) in Experiments 1 and 2

\begin{tabular}{ccc}
\hline $\begin{array}{c}\text { Experi- } \\
\text { ment }\end{array}$ & $\begin{array}{c}\text { One-Letter } \\
\text { Displays }\end{array}$ & $\begin{array}{c}\text { Two-Letter } \\
\text { Displays }\end{array}$ \\
\hline 1 & .25 & .28 \\
2 & .22 & .32 \\
\hline
\end{tabular}

$K$ and $L$, in that all consist of straight lines and angles. The feature-specific interactive channels model would seem to require that performance would be poorer when the target letter was presented with one of the noise letters, $K$ or $L$, than when the single letter occurred alone in the display. However, in only one of the four possible comparisons was performance on the single-target display superior to performance on the corresponding target/nontarget letter display. Again, though, this comparison is clouded by the difference in false-alarm rates for one- and two-letter displays.

Although the data obtained in Experiments 1 and 2 are consistent with the simple hypothesis that the discriminability of a compound stimulus is that of the most discriminable member in the compound, there is good reason to believe that this is not a correct reflection of the underlying perceptual processing. With the identical- and alternative-target displays, the subject has two chances to detect the presence of a target in a display. Based upon the results from the redundant-target experiments summarized above, we should expect a redundancy gain with two target displays. Examination of the data in Table 2 shows that, with only one exception, the identical-target displays have a higher proportion of correct responses than do the corresponding single-target displays. But, again, this comparison is confounded by the higher false-alarm rate for two-letter displays in Experiment 2. This differential false-alarm rate, however, does not confound the comparison between identical and alternative-target displays with target/ nontarget displays. The finding that alternative- and identical-target displays in both experiments gave significantly superior performance to target/nontarget displays is clear support for a redundancy gain in the present experiments.

A redundancy gain in and of itself does not support or refute the feature-specific model. If one could determine the level to be expected from two independent chances to perceive, the model could be tested. Performance on identical-target displays that was at the level to be expected from perceptual independence would be inconsistent with featurespecific inhibition. To answer the question of whether a diverse input actually enhances perceptual performance, one could also determine whether the performance on alternative-target displays was significantly better than that to be expected from independent chances. Unfortunately, the present experimental paradigms are not suitably designed to determine the question of perceptual independence. The differences in false-alarm rates for one- and twoletter displays in Experiment 2 precludes making this type of comparison.

Even were it not for the differential false-alarmrate problem, a test of the feature-specific model via 
a determination of perceptual independence is a difficult and logically unsatisfactory approach. It is logically unsatisfactory because the conclusion of perceptual independence is the same as having to accept the null hypothesis. A conclusion of perceptual independence is based upon the finding that there is no significant difference between the level of performance predicted on the basis of independent chances and the performance obtained. The approach is methodologically complicated by the fact that nonindependence could obtain for reasons that do not test the feature-specific model. In their astute discussion of perceptual independence, Garner and Morton (1969) distinguish between process and state independence. The feature-specific model speaks to process independence, but it can be difficult to experimentally tease out underlying process independence from state variables. For example, should the subject blink (a state variable) at the moment a double target was flashed, he would miss both opportunities to perceive the stimulus. This would introduce correlated error and result in a lower level of performance than that to be expected from independence, even though true process independence obtained.

\section{EXPERIMENT 3}

If the Bjork and Murray paradigm could be modified so as to handle the response-bias problem and eliminate the postdisplay cuing, it would provide an effective means of testing the feature-specific model. In this paradigm, at least one target is always presented in each display and the subject's discrimination is among the possible targets. It differs from the Santee and Egeth paradigm in that the latter requires a discrimination of target letters from a noise, or nontarget, set. The feature-specific model makes clear predictions as to what the results should be with the Bjork and Murray paradigm. Displays consisting solely of a single target should be superior to multitarget displays, and displays consisting of the target and the alternative target should be superior to displays consisting of identical target letters.

To deal with the response-bias problem and the postdisplay cuing, we modified the Bjork and Murray paradigm in the following ways. The letters in a display were presented along the circumference of an imaginary circle $1.5 \mathrm{deg}$ of visual angle in diameter and centered on a fixation cross. The target letter occurred either to the right or the left of the fixation point at a position corresponding to 3 or 9 o'clock. On displays in which noise letters were present, the same noise letter occurred above and below the fixation point at positions corresponding to 12 and $6 o^{\prime}$ clock. The letters used were A, E, and N.
For half the subjects, the targets were the uppercase versions of these letters, and for the other half, they were the lowercase versions. There were five experimental conditions. In the no-noise condition, a single letter, $\mathbf{A}, \mathrm{E}$, or $\mathbf{N}$ in upper- or lowercase, depending upon the subject, appeared at either 3 or 9 o'clock. In the identical-noise condition, the target letter that appeared at 3 or 9 o'clock was replicated in both the 12 and 6 o'clock positions (e.g., $A$ at 3 o'clock, with As at 12 and 6 o'clock). In the name-identical condition, the target letter also occurred in the 12 and 60 'clock positions but in the other case (e.g., $A$ at 9 o'clock, as at 12 and 6 o'clock). In the other-target/ same-case, condition, the target letter occurring at the 3 or 9 o'clock position was accompanied by one of the other two target letters in the 12 and 6 o'clock positions and in the same case (e.g., a at 9 o'clock, e at 6 and 12 o'clock). In the other-target/other-case condition, the target letter was accompanied by one of the other target letters in the opposite case in the two noise-letter positions. While this design is comparable to that of Bjork and Murray in the important sense that the subject's task is to discriminate target letters from each other, it differs from their task in the following ways and for the following reasons.

A major difference was our use of three- rather than two-letter discrimination. There were two reasons for this change. The first was that three-letter discrimination permits a more sensitive test for possible effects of extraneous noise letters. It is possible, by manipulating display energy, to set baselevel performance appreciably above chance but still leave sufficient room for performance improvement without running into ceiling effects. Our experience with perceptual recognition tasks in a tachistoscope has shown that percent correct is related to display energy in essentially an ogival function. Throughout the midrange of percent correct, the relationship to display energy is quite linear, but as percent correct approaches 100 a disproportionate amount of display energy is necessary for further improvement. Thus, the energy increase necessary to change performance from $60 \%$ to $70 \%$ is appreciably less than that needed to move from $85 \%$ to $95 \%$ correct.

The second reason was that the three-letter discrimination reduces any effect of a possible response bias, such as that described earlier, and also permits, as will be seen in the results section, an analysis of the data to determine to what extent a response bias may have been present.

The second change in procedure was the elimination of the postcuing employed by Bjork and Murray. In their experiment, the stimulus display was terminated by a marker indicating which of the two presented letters the subject was to identify. Prior research has shown (Averbach \& Corriell, 1961; 
Colegate, Hoffman, \& Eriksen, 1973) that as much as 250 to $300 \mathrm{msec}$ is required to process this cue and make the selection. In the meantime, the information the subject has received from the display must be held in some form of postexposure storage, where it is subject to decay. With our procedure, the random presentation of the target in either the 3 or 9 o'clock position, which the subject knew beforehand were the only possible target positions, insured central fixation and eliminated the complicating variable of poststimulus storage. It also served to remove, to a large extent, the problem of positional uncertainty as to which letter had occupied which position. Furthermore, and most importantly, the clear delineation of target and nontarget positions eliminated the previously discussed phenomenal probability problem that could induce or reinforce a response bias in the subject.

The use of both the upper- and lowercase versions of the three target letters was thought to vary the noise and targets over a wider range of similarity. The uppercase versions of the three chosen letters are quite similar, in the sense that they consist of straight lines and angles, whereas their lowercase versions are essentially curves. In addition to being able to compare performance among the five conditions, it is also possible to carry out a more powerful test for the effect of feature overlap by comparing the identical-noise condition and the other-target/samecase condition with the name-identical and othertarget/other-case conditions. This assumes that there is more feature overlap between $A, E$, and $N$ than between any one of these uppercase letters and the lowercase $a, e$, and $n$.

The use of two noise letters would seem to provide a more sensitive test of interference at the feature level. The constellations formed by the three letters on noise-letter displays was also thought to help break up any possible confounding effect that might be due to differences in digram frequency that are accentuated with displays presented along the horizontal meridian.

Finally, pre- and postmasks were not used, since there is nothing apparent in the formulation of the feature-specific model that would restrict the operation of the hypothesized processes only to situations in which masking is present. On the contrary, the use of visual masks can introduce serious confounding that complicates interpretation of results (Eriksen, 1980).

\section{Method}

Subjects. Eight students at the University of Illinois (women) served as paid volunteers. All had normal or corrected-to-normal vision.

Apparatus and Stimull. Two channels of a Scientific Prototype three-channel tachistoscope were used. One channel presented the adaptation and postexposure field and contained a black fixation cross. The other channel was used for the presentation of the stimulus displays. Luminance of the white background for both fields was set at $6.8 \mathrm{~cd} / \mathrm{m}^{2}$. Reaction time was recorded through the use of a voice-activated relay and a Hunter Model 1522 digital Klockounter. Although the reaction time data were gathered, they are not presented in the data analysis due to their extreme variability and, in many instances, long times. The variability in the reaction time data was due to the fact that the instructions to the subjects stressed accuracy rather than speed.

The target letters were $\mathrm{A}, \mathrm{E}$, and $\mathrm{N}$ and their lowercase version. The capitals were Presstype Futura Bold 20-pt black letters. The lowercase versions were in 28-pt in order to have all the letters be approximately equal in size, although there was some slight variation in width. The letters were arranged along the circumference of an imaginary circle, $1.5 \mathrm{deg}$ of visual angle in diameter, projected about the point corresponding to the center of the fixation cross. The target letter always appeared either to the left or to the right of the fixation cross at the 9 or 3 o'clock position. The noise letters appeared above and below (12 and 6 o'clock positions). All letters subtended approximately $.25 \mathrm{deg}$ of visual angle, with a .5-deg intercontour distance. This circular display was used to avoid the retinal locus effects of linear displays, and central fixation was encouraged by random variation between the two target positions.

Fifty-four stimulus cards were constructed for each case of the target sets. Within each 54-card set, the three basic noise target conditions occurred equally often: no-noise condition, similar feature noise (identical noise and other target/same case), and dissimilar features (name identical and other target/other case). All the noise conditions were arranged in a pseudorandom order with equal occurrence of each basic noise condition in each third of a block of trials. The random order was restricted so that no more than three uniform responses or target positions could occur successively.

Procedure. Each subject was tested for eye dominance, and then the nondominant eye was occluded with an insert in the appropriate half of the tachistoscope viewing hood. Four subjects were assigned to the uppercase target condition, and the other four to the lowercase condition. The subjects were instructed to hold the handswitch and initiate the stimulus presentation only when the fixation cross was in good focus. They were told the target letter would always appear either to the left or the right of the fixation cross, and they were to ignore other letters which might occur above and below the fixation cross. They were also told that the target would occur at either location equally often. Accuracy was stressed.

Each subject served in four sessions run on consecutive days. Each session consisted of four blocks of 54 trials each. The first session was a practice session to familiarize the subject with the stimuli and the procedure and to determine an exposure duration for that subject that would yield overall accuracy of $50 \%$ to $75 \%$. During the experiment, adjustments were made in exposure duration following a block of trials in order to maintain this performance level.

This experiment was actually run as an experiment and a replication. The experiment was run on four subjects (two assigned upper- and two assigned lowercase targets) first with R.D. as the experimenter and then again several months later with $Y . Y$. as the experimenter. Both experimenters used the same equipment and followed the same procedure. They worked independently of each other.

\section{Results and Discussion}

The accuracy data were evaluated by a four-way mixed design ANOVA. (The experimenters, noise conditions, case of the target, and subjects). None of the main effects and none of the interactions approached significance $(p>.10)$. In Table 4 , the 
Table 4

Percent Correct Target Identifications Under the Different Noise Conditions as Obtained by the Individual Experimenters

\begin{tabular}{|c|c|c|c|c|c|c|c|}
\hline \multirow[b]{2}{*}{ Condition } & \multirow[b]{2}{*}{ Example } & \multicolumn{3}{|c|}{ Uppercase Target } & \multicolumn{3}{|c|}{ Lowercase Target } \\
\hline & & Y.Y. & R.D. & Mean & Y.Y. & R.D. & Mean \\
\hline No Noise & $\mathbf{A}$ & 66 & 60 & 63 & 63 & 62 & 62 \\
\hline Identical Noise & AAA & 60 & 67 & 64 & 62 & 53 & 58 \\
\hline Name-Identical Noise & $\mathrm{aAa}$ & 58 & 60 & 59 & 64 & 62 & 63 \\
\hline Same-Case Noise & EAE & 65 & 67 & 66 & 65 & 59 & 62 \\
\hline Other-Case Noise & eAe & 63 & 64 & 64 & 66 & 60 & 63 \\
\hline
\end{tabular}

mean percent correct for each of the five experimental conditions is shown both separately for the two experimenters and averaged across experimenters. As can be seen from the table, the maximum differences for the averaged data occur between the name-identical and the other-target/other-case noise conditions with the uppercase targets and between the identical and the name-identical conditions with the lowercase targets. Not only are these differences small and nonsignificant, but they are not the differences to be expected from the featurespecific model.

If we make the plausible assumption that there is greater feature similarity within the group of capital letters $\mathbf{A}, \mathrm{E}$, and $\mathbf{N}$ and within the lowercase counterparts $\mathbf{a}, \mathbf{e}$, and $\mathbf{n}$ than there is between these two groups, then a more powerful test of the featurespecific model is possible from our data. The identicalnoise and other-target/same-case conditions (similar features) were pooled across conditions and across upper- and lowercase target groups and compared with the comparably pooled data from the nameidentical and other-target/other-case conditions (dissimilar features). The mean proportion correct for the similar-feature noise was .625 and .623 for the dissimilar feature noise. Since these values are virtually identical, no significance test was indicated.

In the two-letter experiment of Bjork and Murray (1977) and the replication by Santee and Egeth (1980), a response bias could have produced their results in the following way. On a certain proportion of the trials, the subject may be expected to have perceived only one of the two presented letters. If the subsequent marker indicated the nonperceived letter, the subject's bias may have been to guess the target letter other than the one he had clearly perceived. This bias would spuriously increase the hits on alternative target displays and reduce the hits on identical target displays. In the present three-letter experiment, the effect of such a bias would be reduced by one-half. If the subject on a trial should perceive one of the noise letters but not the letter in the target location, a bias to guess a letter other than the one perceived still gives him an alternative of two letters from which to guess. Furthermore, the use of the threeletter design gives us a method for evaluating to what extent such a bias may have been operating in the present experiment.
For each subject, the error trials involving multiletter displays were analyzed in the following manner. The incorrect responses were classified dichotomously in terms of whether they corresponded with the noise letter present on that trial or the third letter not presented. If a response bias were operating, the nonpresented letter should be given as a response more often than the letter presented as noise. A chi-square value was computed for each of the eight subjects. Since the direction of the relationship varied across subjects, the usual summation of chi-squares for an overall significance test was not appropriate. Instead, the summation of the natural log of the probability associated with each individual chi-square was computed. The resulting sum is distributed as chi-square witn $2 \mathrm{~N}$ (16 in this case) degrees of freedom (Gordon, Loveland, \& Cureton, 1952). The summed chi-square value did not approach significance $(p>.10)$.

The absence of a significant response bias effect in the present data does not exonerate the Bjork and Murray experiment and the Santee and Egeth replications of this possible artifact. The present experiment was designed to avoid teaching or reinforcing a response bias in the subject. As noted in the introduction, the Bjork and Murray experimental arrangement could lead to the phenomenal impression on the subject's part that alternative target displays were much more frequent than same target displays. When the four possible two-target displays (AA, EE, AE, and EA) are used with equal frequency, a subject who was attending to As, for example, would note that when he saw an $\mathbf{A}$ in the display and both display letters were perceived on that trial, the letter accompanying the $\mathbf{A}$ would be two times as likely to be an $E$ as another $A$. The present experiment avoided this problem by using fixed positions in the display for target and noise letters. The subject knew that the target appeared only in the 3 or 9 o'clock position and that the 6 and 12 o'clock positions were noise letters. This eliminated the post display cuing that causes the phenomenal probability problem in the Bjork and Murray design. In our experiment, when a subject sees an $A$, for example, in either of the target positions he experiences, $\mathrm{A}, \mathrm{E}$, and $\mathrm{N}$ equally often as the letters occupying the noise positions.

The outcome of our Experiment 3 is completely negative with respect to the feature-specific model. There is no significant or suggestive evidence that 
single-letter displays are recognized more readily or accurately than multiletter displays, nor is there any indication that the feature similarity between the noise and target letters has an effect upon accuracy of identifying the target. We believe that the discrepancy between our findings and the results from the Bjork and Murray experiment and the Santee and Egeth replication is attributable to the elimination of the response bias possibility in our experimental design. However, there are a number of other ways in which our experiment differs from that of Bjork and Murray's, and these need to be considered as possibilities for the discrepancy in outcomes.

We have already discussed the difference in terms of the fixed location for target and noise letters in the present experiment and the elimination of the postdisplay cuing. These modifications were necessary to avoid contributing to a response bias in the subject. There is nothing that is apparent in the formulation of the feature-specific model that would suggest that these modifications would affect feature-specific inhibition. On the contrary, the elimination of the postdisplay cue would seem to make the experimental arrangement more sensitive in detecting possible feature-specific inhibition. The use of a postdisplay cue requires some form of postperceptual storage while the cue is being perceived and comprehended. By elimination of the postperceptual storage, our experimental arrangement would seem to measure performance more directly reflecting the earlier stages in perceptual processing.

The spacing between the target and noise letters in terms of visual angle might be one source of difference, since it is known that close adjacency of contours affects visual acuity (Flom, Weymouth, \& Kahneman, 1969). The distance between noise and target in our experiment was about twice that employed in the Santee and Egeth replications, but it was well within the separations used by Bjork and Murray. Tests of the feature-specific model need to exercise care that the noise and target have a certain minimal separation. Flom, Weymouth, and Kahneman (1963) found that the detection of the gap in a Landolt $\mathbf{C}$ was impaired if black bars were within a third of a degree of visual angle of the target $C$. Eriksen and Rohrbaugh (1970) found that letters, xs, and black disks were equally effective in impairing letter recognition when spaced $.08 \mathrm{deg}$ of angle in intercontour distance from the target letter. Both Eriksen and Rohrbaugh and Flom et al. found that the interference was eliminated at separations greater than a third of a degree of visual angle. The presence of such interference at these close separations does not necessarily implicate feature detectors. Instead, the phenomenon appears to be more parsimoniously explained in terms of the mechanisms underlying visual acuity and contour formation.

A further difference among the experiments was visual masking. Bjork and Murray, as well as Santee and Egeth in their replications, used a visual mask preceding and following display presentation. We deliberately chose not to do so. The feature-specific model, as formulated, provides no basis for expecting the phenomenon to occur only if visual masks are used in the experimental situation. Bjork and Murray provided no rationale as to why they employed a mask. If the model applies only to masked presentations, then the nature of the mask necessary to obtain the effect must be clearly specified. All masks are not equal, nor is the same mask equally effective in masking different targets or letters. Should alternative-target displays be found to be superior to identical-target displays only when a visual mask is employed, this result would probably give us more information about the nature of visual masking rather than reflect upon the validity of the featurespecific model. The mechanisms of visual masking are still controversial (Felsten \& Wasserman, 1981). Thus, experiments that employ visual masks when the masking itself is not under investigation run the high risk of having the interpretation of their results critically dependent upon assumptions concerning the processes that underlie masking.

\section{REFERENCES}

Averbach, E., \& Coriell, A. A. Short term memory in vision. Bell System Technical Journal, 1961, 40, 309-328.

Biederman, G., \& Checkosky, S. F. Processing redundant information. Journal of Experimental Psychology, 1970, 83, 486490.

Bjork, E. L., \& Murray, J. T. On the nature of input channels in visual processing. Psychological Review, 1977, 84, 472-484.

Colegate, R., Hoffman, J. E., \& Eriksen, C. W. Selective encoding from multielement visual displays. Perception \& Psychophysics, 1973, 14, 217-224.

Egeth, H. E., \& Santee, J. L. Conceptual and perceptual components of interletter inhibition. Journal of Experimental Psychology: Human Perception and Performance, 1981, 7, 506-517.

Eriksen, C. W. Independence of successive inputs and uncorrelated error in visual form perception. Journal of Experimental Psychology, 1966, 72, 26-35.

Eriksen, C. W. The use of a visual mask may seriously confound your experiment. Perception \& Psychophysics, 1980, 28, $89-92$.

Eriksen, C. W., \& Collins, J. Temporal course of selective attention. Journal of Experimental Psychology, 1969, 80, 254261.

Eriksen, C. W., \& Greenspon, T. S. Binocular summation over time in the perception of form at brief durations. Journal of Experimental Psychology, 1968, 76, 331-336.

Eriksen, C. W., Greenspon, T. S., Lappin, J. S., \& Carlson, W. A. Binocular summation in the perception of form at brief durations. Perception \& Psychophysics, 1966, 1, 415-441.

Eriksen, C. W., \& Lappin, J. S. Internal perceptual system noise and redundancy in simultaneous inputs in form identification. Psychonomic Science, 1965, 2, 251-352.

Erixsen, C. W., \& LAPPIN, J. S. Independence in the perception of simultaneously presented forms at brief duration. Journal of Experimental Psychology, 1967, 73, 468-472.

Eriksen, C. W., \& Rohrbaugh, J. Some factors determining efficiency of selective attention. American Journal of Psychology, $1970,83,330-342$. 
Eriksen, C. W., \& Schultz, D. W. Information processing in visual search: A continuous flow conception and experimental results. Perception \& Psychophysics, 1979, 25, 249-263.

Eriksen, C. W., \& Spencer, T. Rate of information processing in visual perception: Some results and methodological considerations. Journal of Experimental Psychology Monographs, 1969, 79(No. 2, Pt. 2).

Estes, W. K. Interactions of signals and background variables in visual processing. Perception \& Psychophysics, 1972, 12, 278-286.

Felsten, G., \& Wasserman, G. S. Visual masking: Mechanisms and theories. Psychological Bulletin, 1981, in press.

Flom, M. C., Weymouth, F. W., \& Kahneman, D. Visual resolution and contour interaction. Journal of the Optical Society of America, 1963, 53, 1026-1032.

Gardner, G. T. Evidence for independent parallel channels in tachistoscopic perception. Cognitive Psychology, 1973, 4, 130155.

Garnen, W. R., \& Morton, J. Perceptual independence: Definitions, models and experimental paradigms. Psychological Bulletin, 1969, 72, 233-259.
GiLINSKY, A. S. Orientation-specific effects of adapting light on visual acuity. Journal of the Optical Society of America, 1968, 58, 13-18.

Gordon, M. H., Loveland, E. H., \& Cureton, E. E. An extended table for chi-square for two degrees of freedom, for use in combining probabilities from independent samples. Psycho metrika, 1952, 17, 311-316.

Humphreys, L. G. Acquisition and extinction of verbal expectations in a situation analogous to conditioning. Journal of $E x$ perimental Psychology, 1939, 25, 294-301.

Santev, J. L., \& Eaeth, H. E. Interference in letter identification: A test of feature-specific inhibition. Perception \& Psychophysics, 1980, 27, 321-330.

Shiffrin, R. M., \& Geigler, W. A. Visual recognition in a theory of information processing. In $\mathbf{R}$. L. Solso (Ed.), Contemporary issues in cognitive psychology: The Loyola Symposium. Washington, D.C: Winston, 1973.

(Manuscript received March 16, 1981; revision accepted for publication July 20,1981 .) 